\title{
Role of visual assessment of chronic obstructive pulmonary disease on chest CT: beauty is in the eye of the beholder
}

\author{
Esther Pompe, Firdaus A. A. Mohamed Hoesein \\ Department of Radiology, University Medical Center Utrecht, Utrecht, The Netherlands \\ Correspondence to: Esther Pompe. Department of Radiology, University Medical Center Utrecht, Postbus 85500, 3508 GA Utrecht, The Netherlands. \\ Email: e.pompe@umcutrecht.nl.
}

Submitted Sep 22, 2021. Accepted for publication Nov 12, 2021.

doi: $10.21037 /$ jtd-21-1527

View this article at: https://dx.doi.org/10.21037/jtd-21-1527

Cigarette smoking remains common around the world and is a well-known cause of functional lung impairment, mainly chronic obstructive pulmonary disease (COPD). Currently, COPD is the third cause of death worldwide and responsible for approximately $6 \%$ of total deaths (1). Spirometry is required to establish the diagnosis and severity of COPD, but pulmonary function tests (PFT) have some important limitations: technical difficulties in patients with respiratory problems, relative insensitivity at the early stage of disease and the inability to distinguish subtypes of COPD or provide regional information $(2,3)$.

COPD is considered a complex heterogeneous disease in which disease presentation and course can vary greatly between individual patients and over time. Indeed, despite a similar degree of airflow limitation, patients can present with different clinical outcomes such as respiratory symptoms and exacerbations (4). As a consequence, many studies have been performed in order to identify subtypes of COPD, i.e., phenotypes. PFT results alone may not be sufficient to capture these phenotypes, as the amount of emphysema, small airway disease, and bronchial inflammation cannot be distinguished.

Computed tomography (CT) is an established imaging technique that can be used to capture multiple characteristics of COPD in a non-invasive manner $(5,6)$. By using CT, global and regional information can be obtained. A radiologist can perform radiologic characterization visually by qualitatively scoring several characteristics of COPD. A guideline to standardize and visually categorize patterns of emphysema, airway disease, and associated features has been published by the Fleischner society (7). In this statement, emphysema is categorized as centrilobular emphysema (subclassified as trace, mild, moderate, confluent, and advanced destructive), paraseptal emphysema (PSE, subclassified as mild or substantial), or panlobular emphysema. In the absence of emphysema, airway disease can be a dominant feature of COPD and can be expressed as thickening of bronchial walls, micronodular peripheral opacities, or by gas trapping on expiratory CT.

Visual assessment of emphysema has been associated with impaired lung function, higher risk of mortality, genetic loci associated with COPD, and lung cancer (8-12). However, visual analysis is time consuming and subjective. Alternatively, quantitative assessment can be performed. This may be helpful in providing an objective measure for risk stratification and can be used to analyze large datasets of CT scans, for example in lung cancer screening programs $(9,13,14)$. Multiple studies have been performed to evaluate the most paramount CT-features, but up until now, CT quantification techniques are not widely used in clinical setting.

Recently, Kang et al. evaluated the relationship between visually assessed CT subtypes of COPD with quantitative CT characteristics and clinical traits in a recently published study in the Fournal of Thoracic Disease (15). They included 452 participants from the Chronic Obstructive Pulmonary Disease in Dusty Areas (CODA) cohort, which includes Korean COPD patients living near cement plants. They visually categorized patients into 7 subtypes: normal, PSE, bronchial airway disease (and absent or trace emphysema or mild PSE), trace centrilobular emphysema, mild centrilobular emphysema, moderate centrilobular emphysema, and confluent and advanced destructive emphysema. 
The results of Kang et al. confirmed previously suggested associations: (I) body mass index (BMI) was lower in patients with a higher degree of emphysema and relatively higher in the bronchial airway group; (II) pulmonary function lowered as the degree of emphysema worsened; (III) Pi10 was highest in the bronchial disease subtype; (IV) emphysema and functional small airways disease as assessed by parametric response mapping $\left(\mathrm{PRM}^{\mathrm{Emph}}\right.$ and $\mathrm{PRM}^{\mathrm{fSAD}}$, respectively) were raised as the visual grade of emphysema worsened; (V) $\mathrm{PRM}^{\mathrm{Emph}}$ increased with severity of emphysema subtypes; (VI) PRM ${ }^{\mathrm{fSAD}}$ was increased in the trace emphysema group as compared to the normal group and decreased slightly in the mild emphysema group.

The PSE subtype was explored in more detail. Participants with PSE had lower lung function and higher PRM ${ }^{\text {Emph }}$ as compared to participants with moderate centrilobular emphysema. PRM ${ }^{\mathrm{fSAD}}$ was lowest in participants with PSE. PSE was categorized regardless of other imaging characteristics, thus participants in the PSE subcategory could have centrilobular emphysema or bronchial airway thickening as well.

Kang and colleagues force us to take a step back and contemplate the value of automatic quantification. As stated before, many studies have been published on automatic quantification techniques that are able to assess several traits of COPD. But why are pulmonologists and radiologists apprehensive for using it in clinical practice?

Already in 1978, shortly after the introduction of CT, the first quantitative analysis of lung density was published (16). Typical histograms for inspiration and expiration were presented and offered the possibility to obtain quantitative information from lung CT. The method was demonstrated to be reproducible, but the authors already denoted limitations caused by artifacts and incomplete inspiration. In almost 40 years, these limitations still play an important role. Moreover, the introduction of multiple scanning techniques, reconstruction filters, scanner models and manufacturers did not improve this either. As a result, the quantification of emphysema (but also air trapping), which is still mainly based on thresholding, is subject to a great number of variables.

The main inconvenience in lung quantification originates from the organ itself; the lung is not a homogeneous substance, but a structured organ with changing density values caused by different vascular, interstitial, and air portions. Lung density can decrease with an increase in the amount of alveolar space (as in emphysema), but can also increase with increasing interstitial space (i.e., in fibrosis). Both processes can appear in the same damaged lung, which indicates the need for regional information.

A second difficulty of the lung that no other organ of the human body does, is that its density changes in relation to its function. With maximum inspiration and expiration, a striking alteration of lung density values is seen, which makes it necessary to standardize the functional condition of the lung during CT acquisition in order to achieve reproducible values. As reproducing maximum inspiration seems to be fairly accessible, exhaling at a constant maximum degree is less well reproducible (17).

Will artificial intelligence (AI) amplify the introduction of quantitative CT analyses in clinical practice? Probably. AI enables more precise segmentation of fissures, lobes, and the broncho-vascular tree, which is an essential step for image quantification $(18,19)$. AI can help in detecting early parenchymal injury before irreversible damage appears whereby knowledge on early parenchymal changes in COPD can be acquired. By linking this to genetic loci, functional data on the genetic and molecular basis of COPD will be enabled (20).

Although AI systems are mainly trained with human labels, AI delivers beyond human expertise as it comes to recognition tasks and classification performance (21). AI can interpret data without prior hypotheses and can explore new phenotypes by connecting imaging phenotypes to genetic and molecular features. This however leads to more empirical data and not to implementation in clinical use. A challenge with $\mathrm{AI}$ is that it is trained with specific samples of a specific population and further replication studies are needed to define its true performance in clinical practice. This requires careful data sharing infrastructures that preserve data integrity and privacy. Another challenge includes our urge to understand AI approaches and the dislike of the 'black box', although this is not always grounded as parameters are available and can be inspected.

Quantitative CT evaluation can successfully identify emphysema, expiratory airflow obstruction, airway wall thickening, and measure lung movement by using dynamicventilation CT, but perhaps this does not fully capture the information available from visual subtyping and other smoking related lung changes. Visually, radiologists can encounter quality issues and look beyond noise, reconstruction filter, artifacts, etc. In addition, a radiologist takes into consideration the patients' clinical traits and history. Subtypes have been defined with visual but no quantitative 
emphysema, which could be a result of regional emphysema or an accompanying increase in lung density, highlighting the importance of a radiologists' evaluation (22). A drawback of visual scoring is its subjectivity and need for experience. One study showed that visual assessment of the presence of COPD on a chest CT is less sensitive than PFT (23). Considering all things, PFT, visual and quantitative CT evaluation are currently regarded as complementary methods to assess COPD.

For now, as it comes to the assessment of COPD phenotypes, beauty is in the eye of the beholder. To be a beholder, one has to pay attention and be aware of other helpful techniques. In other words, the beauty does not exist on its own but is created by the observer.

\section{Acknowledgments}

Funding: None.

\section{Footnote}

Provenance and Peer Review: This article was commissioned by the editorial office, fournal of Thoracic Disease. The article has undergone external peer review.

Peer Review File: Available at https://dx.doi.org/10.21037/ jtd-21-1527

Conflicts of Interest: Both authors have completed the ICMJE uniform disclosure form (available at https://dx.doi. org/10.21037/jtd-21-1527). EP reports that she received a consultancy fee from Thirona BV in 2019. The other author has no conflicts of interest to declare.

Ethical Statement: The authors are accountable for all aspects of the work in ensuring that questions related to the accuracy or integrity of any part of the work are appropriately investigated and resolved.

Open Access Statement: This is an Open Access article distributed in accordance with the Creative Commons Attribution-NonCommercial-NoDerivs 4.0 International License (CC BY-NC-ND 4.0), which permits the noncommercial replication and distribution of the article with the strict proviso that no changes or edits are made and the original work is properly cited (including links to both the formal publication through the relevant DOI and the license). See: https://creativecommons.org/licenses/by-nc-nd/4.0/.

\section{References}

1. World Health Organization. Projections of Mortality and Causes of Death. Date last updated: June 2021.

2. Tantucci C, Modina D. Lung function decline in COPD. Int J Chron Obstruct Pulmon Dis 2012;7:95-9.

3. Agusti A, Edwards LD, Celli B, et al. Characteristics, stability and outcomes of the 2011 GOLD COPD groups in the ECLIPSE cohort. Eur Respir J 2013;42:636-46.

4. Han MK, Agusti A, Calverley PM, et al. Chronic obstructive pulmonary disease phenotypes: the future of COPD. Am J Respir Crit Care Med 2010;182:598-604.

5. Galbán CJ, Han MK, Boes JL, et al. Computed tomography-based biomarker provides unique signature for diagnosis of COPD phenotypes and disease progression. Nat Med 2012;18:1711-5.

6. Hoff BA, Pompe E, Galbán S, et al. CT-Based Local Distribution Metric Improves Characterization of COPD. Sci Rep 2017;7:2999.

7. Lynch DA, Austin JH, Hogg JC, et al. CT-Definable Subtypes of Chronic Obstructive Pulmonary Disease: A Statement of the Fleischner Society. Radiology 2015;277:192-205.

8. Smith BM, Austin JH, Newell JD Jr, et al. Pulmonary emphysema subtypes on computed tomography: the MESA COPD study. Am J Med 2014;127:94.e7-23.

9. Pompe E, de Jong PA, Lynch DA, et al. Computed tomographic findings in subjects who died from respiratory disease in the National Lung Screening Trial. Eur Respir J 2017;49:1601814.

10. Lynch DA, Moore CM, Wilson C, et al. CT-based Visual Classification of Emphysema: Association with Mortality in the COPDGene Study. Radiology 2018;288:859-66.

11. Halper-Stromberg E, Cho MH, Wilson C, et al. Visual Assessment of Chest Computed Tomographic Images Is Independently Useful for Genetic Association Analysis in Studies of Chronic Obstructive Pulmonary Disease. Ann Am Thorac Soc 2017;14:33-40.

12. Wilson DO, Weissfeld JL, Balkan A, et al. Association of radiographic emphysema and airflow obstruction with lung cancer. Am J Respir Crit Care Med 2008;178:738-44.

13. Mets OM, Buckens CF, Zanen P, et al. Identification of chronic obstructive pulmonary disease in lung cancer screening computed tomographic scans. JAMA 2011;306:1775-81.

14. Labaki WW, Xia M, Murray S, et al. Quantitative Emphysema on Low-Dose CT Imaging of the Chest and Risk of Lung Cancer and Airflow Obstruction: An 
Analysis of the National Lung Screening Trial. Chest 2021;159:1812-20.

15. Kang HS, Bak SH, Oh HY, et al. Computed tomographybased visual assessment of chronic obstructive pulmonary disease: comparison with pulmonary function test and quantitative computed tomography. J Thorac Dis 2021;13:1495-506.

16. Wegener OH, Koeppe P, Oeser H. Measurement of lung density by computed tomography. J Comput Assist Tomogr 1978;2:263-73.

17. Mets OM, Isgum I, Mol CP, et al. Variation in quantitative CT air trapping in heavy smokers on repeat CT examinations. Eur Radiol 2012;22:2710-7.

18. Gerard SE, Patton TJ, Christensen GE, et al. FissureNet: A Deep Learning Approach For Pulmonary Fissure Detection in CT Images. IEEE Trans Med Imaging 2019;38:156-66.

Cite this article as: Pompe E, Mohamed Hoesein FAA. Role of visual assessment of chronic obstructive pulmonary disease on chest CT: beauty is in the eye of the beholder. J Thorac Dis 2021;13(12):6936-6939. doi: 10.21037/jtd-21-1527
19. Yun J, Park J, Yu D, et al. Improvement of fully automated airway segmentation on volumetric computed tomographic images using a 2.5 dimensional convolutional neural net. Med Image Anal 2019;51:13-20.

20. Parker MM, Hao Y, Guo F, et al. Identification of an emphysema-associated genetic variant near TGFB2 with regulatory effects in lung fibroblasts. Elife 2019;8:42720.

21. Humphries SM, Notary AM, Centeno JP, et al. Deep Learning Enables Automatic Classification of Emphysema Pattern at CT. Radiology 2020;294:434-44.

22. Park J, Hobbs BD, Crapo JD, et al. Subtyping COPD by Using Visual and Quantitative CT Imaging Features. Chest 2020;157:47-60.

23. Mets OM, Smit EJ, Mohamed Hoesein FA, et al. Visual versus automated evaluation of chest computed tomography for the presence of chronic obstructive pulmonary disease. PLoS One 2012;7:e42227. 